\title{
Post-Extubation Inhaled Nitric Oxide Therapy via High-Flow Nasal Cannula After Fontan Procedure
}

\author{
Yuji Tominaga ${ }^{1}$ (D) Shigemitsu Iwai ${ }^{1}$ - Sanae Yamauchi ${ }^{1} \cdot$ Miyako Kyogoku ${ }^{2} \cdot$ Yosuke Kugo $^{1} \cdot$ Moyu Hasegawa $^{1}$. \\ Futoshi Kayatani $^{3} \cdot$ Kunihiko Takahashi $^{3} \cdot$ Hisaaki Aoki $^{3} \cdot$ Muneyuki Takeuchi $^{2} \cdot$ Kazuya Tachibana $^{4} \cdot$ Hiroaki Kawata $^{1}$
}

Received: 13 March 2019 / Accepted: 2 May 2019 / Published online: 7 May 2019

(c) Springer Science+Business Media, LLC, part of Springer Nature 2019

\begin{abstract}
In 2014, our hospital introduced inhaled nitric oxide (iNO) therapy combined with high-flow nasal cannula (HFNC) oxygen therapy after extubation following the Fontan procedure in patients with unstable hemodynamics. We report the benefits of HFNC-iNO therapy in these patients. This was a single-center, retrospective review of 38 patients who underwent the Fontan procedure between January 2010 and June 2016, and required iNO therapy before extubation. The patients were divided into two groups: patients in Epoch $1(\mathrm{n}=24)$ were treated between January 2010 and December 2013, receiving only iNO therapy; patients in Epoch $2(n=14)$ were treated between January 2014 and June 2016, receiving iNO therapy and additional HFNC-iNO therapy after extubation. There were no significant differences between Epoch 1 and 2 regarding preoperative cardiac function, age at surgery, body weight, initial diagnosis (hypoplastic left heart syndrome, 4 vs. 2; total anomalous pulmonary venous return, 5 vs. 4 ; heterotaxy, 7 vs. 8), intraoperative fluid balance, or central venous pressure upon admission to the intensive care unit. Epoch 2 had a significantly shorter duration of postoperative intubation [7.2 (3.7-49) vs. 3.5 (3.0-4.6) hours, $p=0.033$ ], pleural drainage [23 (13-34) vs. 9.5 (8.3-18) days, $p=0.007$ ], and postoperative hospitalization [36 (29-49) vs. 27 (22-36) days, $p=0.017$ ]. Two patients in Epoch 1 (8.3\%), but none in Epoch 2, required re-intubation. Our results suggest that HFNC-iNO therapy reduces the duration of postoperative intubation, pleural drainage, and hospitalization.
\end{abstract}

Keywords Inhaled nitric oxide $\cdot$ High-flow nasal cannula $\cdot$ Fontan operation $\cdot$ Pleural drainage $\cdot$ Hospitalization

Presented at the 7th World Congress of Pediatric Cardiology and Pediatric Surgery, Barcelona, Spain, July 16-21, 2017.

Yuji Tominaga

ytomtomy@gmail.com

1 Department of Cardiovascular Surgery, Osaka Women's and Children's Hospital, 840 Murodocho, Izumi 594-1101, Osaka, Japan

2 Department of Intensive Care Medicine, Osaka Women's and Children's Hospital, 840 Murodocho, Izumi 594-1101, Osaka, Japan

3 Department of Pediatric Cardiology, Osaka Women's and children's Hospital, 840 Murodocho, Izumi 594-1101, Osaka, Japan

4 Department of Anesthesiology, Osaka Women's and Children's Hospital, 840 Murodocho, Izumi 594-1101, Osaka, Japan

\section{Introduction}

Maintaining low pulmonary vascular resistance after the Fontan procedure is important. Inhaled nitric oxide (iNO) is a selective pulmonary vasodilator that decreases pulmonary arterial pressure and pulmonary vascular resistance without inducing systemic hemodynamic effects. iNO therapy during intubation has been shown to be effective in the postoperative management of high-risk Fontan candidates [1-5]. Discontinuation of iNO, however, often leads to a "rebound" phenomenon, which involves systemic hypotension and pulmonary hypertension. Furthermore, there is no standardized protocol for continuing iNO therapy after extubation. In such cases, prolonged intubation and sometimes re-intubation are necessary. Sildenafil was reportedly used for persistent pulmonary hypertension and for prevention of the "rebound" phenomenon early after congenital cardiac surgery in children [6,7], but this agent is not pulmonary selective and induces hypotension as a systemic hemodynamic side effect. Moreover, the effect of sildenafil 
is unpredictable and depends on its degree of absorption. Gastrointestinal absorption is known to be insufficient, particularly in patients with high central venous pressure after undergoing the Fontan operation. Delivering iNO therapy using a high-flow nasal cannula (HFNC) has been reported in infants with pulmonary hypertension and respiratory distress $[8,9]$. This approach permits continuation of iNO therapy after extubation. Few reports have described the use of iNO therapy delivered via an HFNC (HFNC-iNO therapy) following extubation during postoperative management for congenital heart disease. In 2014, our hospital introduced the use of HFNC-iNO therapy after extubation following the Fontan procedure. In this retrospective study, we investigated the benefits of HFNC-iNO in these patients.

\section{Materials and Methods}

\section{Patients and Study Design}

Permission to conduct this study and publish the outcomes was provided by the Institutional Review Board of our institution. Written informed consent for the use of patient records was obtained from the legal guardian of each patient. HFNC-iNO therapy after extubation was introduced in our hospital's practice in January 2014 and has since been applied to 14 patients with high pulmonary artery pressure and low cardiac output after the Fontan operation. We compared them to the patients who underwent only iNO therapy during intubation before introduction of HFNC-iNO therapy. This single-center, retrospective study consisted of reviewing the records of 52 consecutive patients who underwent the Fontan operation at our hospital between January 2010 and June 2016, and received iNO therapy in the operating room and/or intensive care unit (ICU) before extubation. The following 14 patients were excluded: patients aged $>15$ years $(n=2)$, patients with a single lung $(n=1)$, patients with preoperative tracheostomy $(n=1)$, patients who underwent atrioventricular valve plasty due to moderate-to-severe valve regurgitation $(n=4)$, patients with postoperative phrenic nerve paralysis $(n=4)$, patients with postoperative tracheal bleeding $(n=1)$, and patients with postoperative cerebral complications $(n=1)$. Finally, 38 patients were included in the study. We divided the patients into two groups according to whether they were treated before or after the change in treatment protocol: patients in Epoch $1(n=24)$ were treated between January 2010 and December 2013, receiving only iNO therapy during intubation; patients in Epoch $2(n=14)$ were treated between January 2014 and June 2016, receiving iNO therapy during intubation and additional HFNC-iNO therapy after extubation. The same two operators performed the Fontan operations in all patients. The rest of the treatment protocol (operation, extubation, and postoperative management) did not differ between the two studied periods. The two groups were compared in terms of perioperative outcomes.

Demographic characteristics such as age, weight, sex, and preoperative cardiac diagnosis were recorded. Preoperative cardiac catheterization and echocardiography results were examined to obtain data regarding the pulmonary artery pressure and cardiac function. Ventricular dominance was defined as left, right, and mixed (e.g., unbalanced common atrioventricular canal). For the mixed-morphology group, the volume of each ventricle was measured separately, and the combined values were used for data analysis. We defined the grade of common atrioventricular valve regurgitation as none, 0 ; trivial, 1 ; mild, 2 ; moderate, 3 ; or severe, 4 . Operative records were reviewed to obtain data regarding the surgical procedure and fluid balance. Postoperative ICU charts were reviewed to obtain hemodynamic parameters, lactate levels, the ratio of arterial oxygen partial pressure to fractional inspired oxygen, the partial pressure of carbon dioxide in arterial blood, the catecholamine score, urine output, and infusion volume. The catecholamine score was calculated as the sum of the levels of all catecholamines, corrected for potency: $1 \times$ dopamine $(\mu \mathrm{g} / \mathrm{kg} / \mathrm{min})+1 \times$ dobutamine $(\mu \mathrm{g} / \mathrm{kg} /$ $\mathrm{min})+100 \times$ epinephrine $(\mu \mathrm{g} / \mathrm{kg} / \mathrm{min})+100 \times$ norepinephrine $(\mu \mathrm{g} / \mathrm{kg} / \mathrm{min})$. Postoperative pulmonary artery pressure was measured in artificially ventilated patients with a positive end-expiratory pressure of $5 \mathrm{~cm} \mathrm{H}_{2} \mathrm{O}$. Postoperative infusion volume included colloids, crystalloids, medications, and blood products. The duration of pleural drainage was defined as the number of days until the pleural drainage rate reduced to below $3 \mathrm{~mL} / \mathrm{kg} / \mathrm{day}$. The main outcomes analyzed were the number of re-intubations and the duration of postoperative intubation, pleural drainage, and postoperative hospital stay, which were compared between the two groups.

\section{Indications for iNO Therapy}

The indication for initiation of iNO therapy was based on the clinical judgement of the attending surgeon, anesthesiologists, and intensivists, and included low blood pressure (systolic blood pressure $<60 \mathrm{mmHg}$ ), high central venous pressure $(>16 \mathrm{mmHg}$ ), and low urine output (less than $0.5 \mathrm{ml} / \mathrm{kg} / \mathrm{h}$ ) after a failed attempt to improve hemodynamics by adjusting intravascular volume and inotrope dose. The patients were weaned off iNO as soon as they fulfilled above-mentioned criteria (i.e., concerning blood pressure, central venous pressure, and urine output).

\section{Protocol for HFNC Therapy and iNO Therapy}

iNO therapy was started in the operating room or ICU. All patients were mechanically ventilated using synchronized intermittent mandatory ventilation with pressure control 
and pressure support mode, using a Puritan Bennett ${ }^{\mathrm{TM}} 840$ ventilator (Puritan Bennett, Pleasanton, CA, USA). The patients were initially ventilated with a positive end-expiratory pressure of $5 \mathrm{~cm} \mathrm{H}_{2} \mathrm{O}$ and $8-10 \mathrm{~mL} / \mathrm{kg}$ tidal volume breaths. Until December 2015, NO gas was administered from stock cylinders containing $800 \mathrm{ppm}$ NO balanced with nitrogen gas (Sumitomo Seika Chemicals Co., LTD., Tokyo, Japan), and delivered to the patient as a continuous flow into the inspiratory limb of the ventilator circuit close to the endotracheal tube. From January 2016, we used the INOvent device (Air Water Inc., Clinton, NJ, USA) for delivering iNO therapy in a similar manner. Patients were initially placed on iNO therapy at $20 \mathrm{ppm}$. The concentration of iNO was rapidly weaned, and the patients were extubated soon after initial stabilization and improvement in systemic perfusion, to encourage spontaneous ventilation. If iNO could not be weaned during intubation, we continued iNO therapy after extubation, using an Optiflow ${ }^{\mathrm{TM}}$ Nasal High Flow system (Fisher \& Paykel Healthcare, Auckland, New Zealand), at a flow rate of $2 \mathrm{~L} / \mathrm{kg} / \mathrm{min}$, fraction of inspired oxygen of $0.5-0.8$, and iNO concentration of $1-5 \mathrm{ppm}$.
Oxygen, air, and $\mathrm{NO}$ were connected to the gas delivery circuit and administered via the HFNC as a mixed gas. If possible, patients were weaned from iNO. If necessary, patients were started on oral phosphodiesterase $\mathrm{V}$ inhibitor. In patients with hemodynamic instability after extubation, we used HFNC-iNO therapy before attempt of re-intubation. After completing the iNO therapy, patients were weaned from HFNC oxygen therapy as soon as possible, and oxygen therapy via a regular nasal cannula was started. $\mathrm{NO}$ and $\mathrm{NO}_{2}$ concentrations were measured from a Lurelock adaptor (Fisher \& Paykel Healthcare, Auckland, New Zealand) inserted in the circuit near the connector to the Optiflow ${ }^{\mathrm{TM}}$ Junior Nasal Cannula (Fisher \& Paykel Healthcare, Auckland, New Zealand) (Fig. 1). During iNO treatment, methemoglobin levels were checked every 3-6 h.

\section{Statistical Analysis}

Statistical analysis was performed using JMP Pro version 14 (SAS Institute Inc., Cary, NC, USA). Data were described as the mean \pm standard deviation or median [interquartile

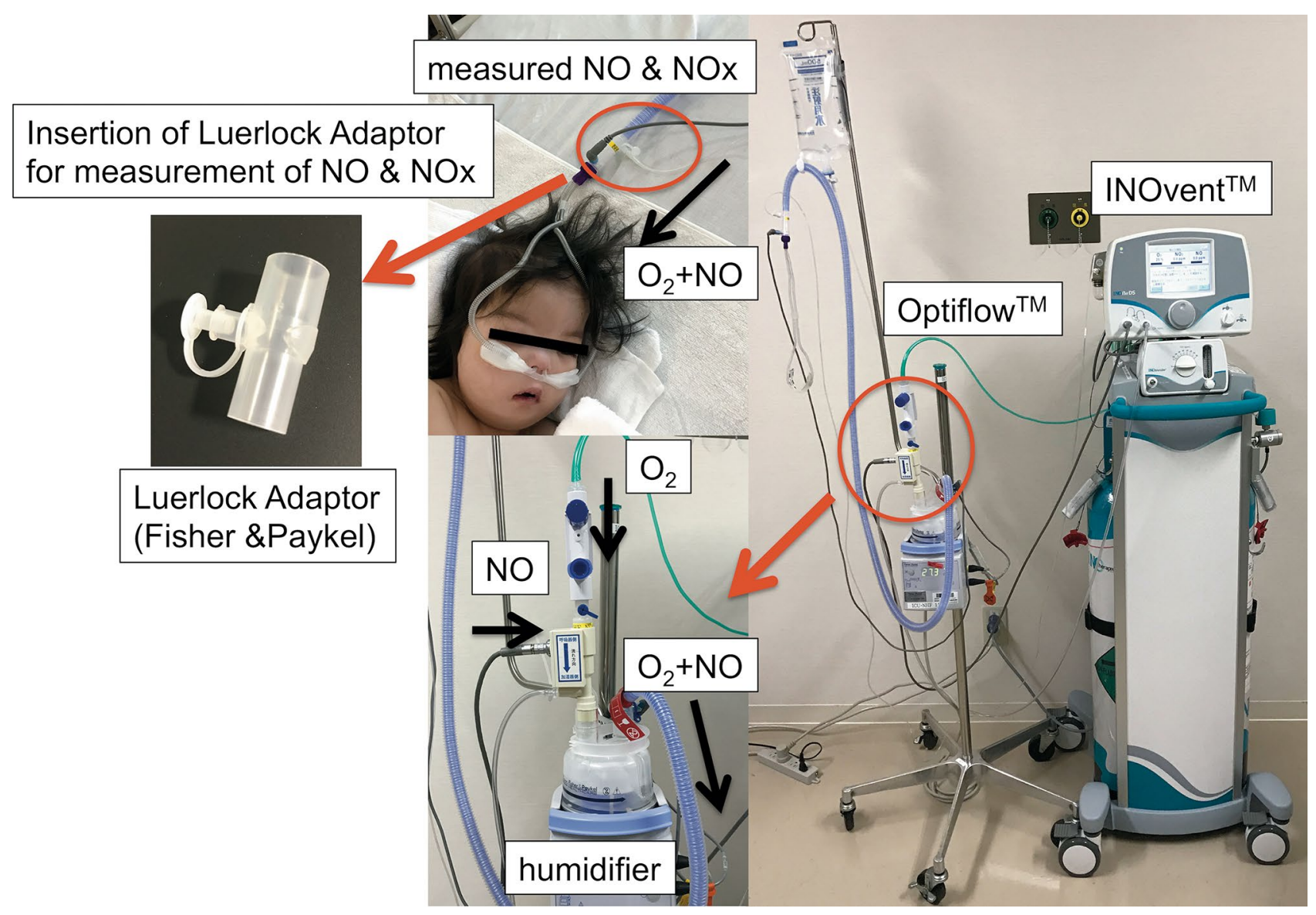

Fig. 1 Setup for combining inhaled nitric oxide therapy with oxygen therapy using the same high-flow nasal cannula circuit. The iNO vent circuit was connected to the Optiflow ${ }^{\mathrm{TM}}$ Nasal High Flow system before the humidifier. A Lurelock adaptor was inserted in the circuit just before the connector to the Optiflow ${ }^{\mathrm{TM}}$ Junior Nasal Cannula for measuring nitric oxide and nitric dioxide concentrations 
range]. Categorical variables were analyzed using the Pearson chi-square test or Fisher exact test, as appropriate. Continuous variables with a normal distribution and continuous variables with non-normal distribution were analyzed using the Student $t$ test and Man-Whitney $U$ test, respectively. Statistical significance was set at a probability value of $<0.05$.

\section{Results}

\section{Patients' Characteristics and Intraoperative Data}

There were no significant between-group differences in body weight $(11.7 \pm 2.3$ vs. $12.2 \pm 1.9 \mathrm{~kg} ; p=0.53)$ and age $(3.5 \pm 1.2$ vs. $2.7 \pm 1.0$ years; $p=0.055)$ between Epoch 1 and Epoch 2 at the time of the Fontan operation. The distribution of diagnoses was also similar between Epoch 1 and 2: hypoplastic left heart syndrome and its variants (4 vs. 2 patients), total anomalous pulmonary venous return ( 5 vs. 4 patients), and heterotaxy syndrome ( 7 vs. 8 patients) (Table 1). Furthermore, the groups were similar in terms of preoperative single-ventricle end-diastolic volume index $\left(143 \pm 44\right.$ vs. $\left.145 \pm 33 \mathrm{~mL} / \mathrm{m}^{2} ; p=0.81\right)$ and pressure $(6.8 \pm 2.1$ vs. $6.6 \pm 3.3 \mathrm{mmHg} ; p=0.78)$,

Table 1 Preoperative characteristics

\begin{tabular}{llll}
\hline Characteristics & Epoch 1 & Epoch 2 & $p$ value \\
\hline Total no. of patients & 24 & 14 & - \\
Males & 6 & 8 & 0.08 \\
Age at Fontan operation (years) & $3.5 \pm 1.2$ & $2.7 \pm 1.0$ & 0.055 \\
BW at Fontan operation (kg) & $11.7 \pm 2.3$ & $12.2 \pm 1.9$ & 0.53 \\
Ventricular dominance & & & - \\
Left ventricle & 4 & 3 & \\
$\quad$ Tricuspid atresia & 3 & 2 & \\
$\quad$ PA/IVS & 1 & 1 & \\
Right ventricle & 14 & 7 & \\
$\quad$ HLHS and HLHS variants & 6 & 2 & \\
$\quad$ Single right ventricle & 4 & 4 & \\
$\quad$ DORV & 4 & 1 & \\
Mixed & 6 & 4 & \\
$\quad$ ccTGA & 3 & 0 & \\
$\quad$ Unbalanced CAVC & 2 & 2 & 0.7 \\
$\quad$ TGA & 1 & 2 & 0.17 \\
TAPVR & 5 & 4 & \\
Pulmonary atresia & 5 & 2 & \\
Heterotaxy & 7 & 8 & \\
\hline
\end{tabular}

Continuous data are presented as the mean \pm standard deviation, while categorical data are shown as the number of observations. $B W$ body weight, $C A V C$ common atrioventricular canal, $c c T G A$ congenitally corrected transposition of great arteries, $H L H S$ hypoplastic left heart syndrome, $P A / I V S$ pulmonary atresia with intact ventricular septum, TAPVR total anomalous pulmonary venous return as well as in terms of ejection fraction $(56 \pm 7 \%$ vs. $53 \pm 6 \% ; p=0.15)$, mean pulmonary pressure $(11 \pm 2 \mathrm{vs}$. $11 \pm 3 \mathrm{mmHg} ; p=0.96)$, PA-index $(261 \pm 112$ vs. $217 \pm 59$ $\mathrm{mm}^{2} / \mathrm{m}^{2} ; p=0.13$ ) [10], and grade of common atrioventricular valve regurgitation. All patients underwent extracardiac total cavopulmonary connection. Concomitant surgical procedures in Epoch 1 and 2 included fenestration (3 vs. 2 patients), Damus-Kaye-Stansel procedure (2 vs. 2 patients), and atrial septal defect enlargement (6 vs. 2 patients). Modified ultrafiltration was performed in all cases. Intraoperative fluid balance did not differ between both groups $(663 \pm 383$ vs. $612 \pm 358 \mathrm{~mL} ; p=0.68)$. iNO therapy was started during the operation in 17 patients in Epoch 1, and in 10 patients in Epoch $2(p=1.0)$ (Table 2).

\section{Postoperative Course of the Fontan Operation}

Upon ICU admission, central venous pressure (14.8 \pm 1.7 vs. $14.4 \pm 1.5 \mathrm{mmHg} ; p=0.47)$, lactate levels $(4.0 \pm 2.3$ vs. $3.8 \pm 1.4 \mathrm{mmol} / \mathrm{L} ; p=0.67)$, creatinine levels $(0.34 \pm 0.09$ vs. $0.32 \pm 0.11 \mathrm{mg} / \mathrm{dL} ; p=0.25)$, the ratio of arterial oxygen partial pressure to fractional inspired oxygen $(234 \pm 109$ vs. $243 \pm 160 ; p=0.84)$, the partial pressure of carbon dioxide in arterial blood $(39 \pm 5$ vs. $40 \pm 7 \mathrm{mmHg}$; $p=0.53)$, and mixed venous oxygen saturation $(63 \pm 14 \%$ vs. $57 \pm 11 \% ; p=0.14$ ) were not significantly different between Epoch 1 and Epoch 2. iNO therapy was started in the ICU in 7 patients in Epoch 1, and in 4 patients in Epoch $2(p=0.52)$. During iNO therapy, methemoglobin levels were below the normal upper limit in all patients.

Compared to Epoch 1, Epoch 2 was characterized by significantly shorter duration of postoperative intubation [7.2 (3.7-49) vs. 3.5 (3.0-4.6) hours, $p=0.033]$. The median duration of HFNC-iNO therapy was 58 [20-102] hours. Two patients in Epoch 1 (8.3\%), but none in Epoch 2, required re-intubation. The urinary output per body weight at $6 \mathrm{~h}$ post-surgery was significantly greater $(p=0.042)$ and the infusion volume per body weight at $24 \mathrm{~h}$ post-surgery was significantly lower $(p<0.01)$ in Epoch 2 than in Epoch 1 (Table 2). Furthermore, the duration of pleural drainage [23 (13-34) vs. 9.5 (8.3-18) days, $p=0.007$ ) and postoperative hospitalization [36 (29-49) vs. 27 (22-33.5) days, $p=0.017]$ were significantly shorter in Epoch 2 than in Epoch 1 (Fig. 2). On the other hand, there was no significant difference between the groups regarding the rates of postoperative catheter or surgical intervention ( 4 vs. $2 ; p=0.85$ ), infection (4 vs. 2 patients; $p=0.85$ ), or chylothorax ( 7 vs. 1 patients; $p=0.11$ ) (Table 3). Upon discharge, 12 patients $(50 \%)$ in Epoch 1, and 9 patients (64\%) in Epoch 2 were prescribed medication for pulmonary hypertension $(p=0.4)$. 
Table 2 Preoperative cardiac function and operative status

\begin{tabular}{llll}
\hline Parameter & Epoch 1 $(n=24)$ & Epoch 2 $(n=14)$ & $p$ value \\
\hline SVEDVI $\left(\mathrm{mL} / \mathrm{m}^{2}\right)$ & $143 \pm 44$ & $145 \pm 33$ & 0.81 \\
SVEDP $(\mathrm{mmHg})$ & $6.8 \pm 2.1$ & $6.6 \pm 3.3$ & 0.78 \\
Ejection fraction $(\%)$ & $56 \pm 7$ & $53 \pm 6$ & 0.15 \\
Mean PAP $(\mathrm{mmHg})$ & $11 \pm 2$ & $11 \pm 3$ & 0.96 \\
PA-index, $\mathrm{mm}^{2} / \mathrm{m}^{2}$ & $261 \pm 112$ & $217 \pm 59$ & 0.13 \\
$<200$ & 13 & 7 & 0.8 \\
CAVVR grade & $1.3 \pm 0.9$ & $1.3 \pm 0.6$ & 0.86 \\
Concomitant surgical procedure & & & - \\
Fenestration & 3 & 2 & \\
Damus-Kaye-Stansel procedure & 2 & 2 & \\
ASD enlargement & 6 & 2 & 0.68 \\
Peripheral PA plasty & 1 & $612 \pm 358$ & 1.0 \\
Fluid balance (mL) & $663 \pm 383$ & 10 & \\
iNO therapy started in OR & 17 & & \\
\hline
\end{tabular}

Continuous data are presented as the mean \pm standard deviation, while categorical data are given as the number of observations. SVEDVI single-ventricle end-diastolic volume index, SVEDP single-ventricle enddiastolic pressure, $P A P$ pulmonary artery pressure, $C A V V R$ common atrioventricular valve regurgitation, $A S D$ atrial septal defect, $P A$ pulmonary artery, $i N O$ inhaled nitric oxide, $O R$ operating room

Fig. 2 Outcomes of the Fontan operation. The patients were divided into two groups according to whether or not HFNCiNO therapy was administered after NO therapy: Epoch 1 $(n=24$; treated between January 2010 and December 2013; received only iNO therapy) vs. Epoch $2(n=14$; treated between January 2014 and June 2016; received iNO therapy and additional HFNC-iNO therapy after extubation). Epoch 2 had a significantly shorter duration of postoperative intubation, pleural drainage, and postoperative hospitalization. HFNC highflow nasal cannula, $i N O$ inhaled nitric oxide, $N O$ nitric oxide
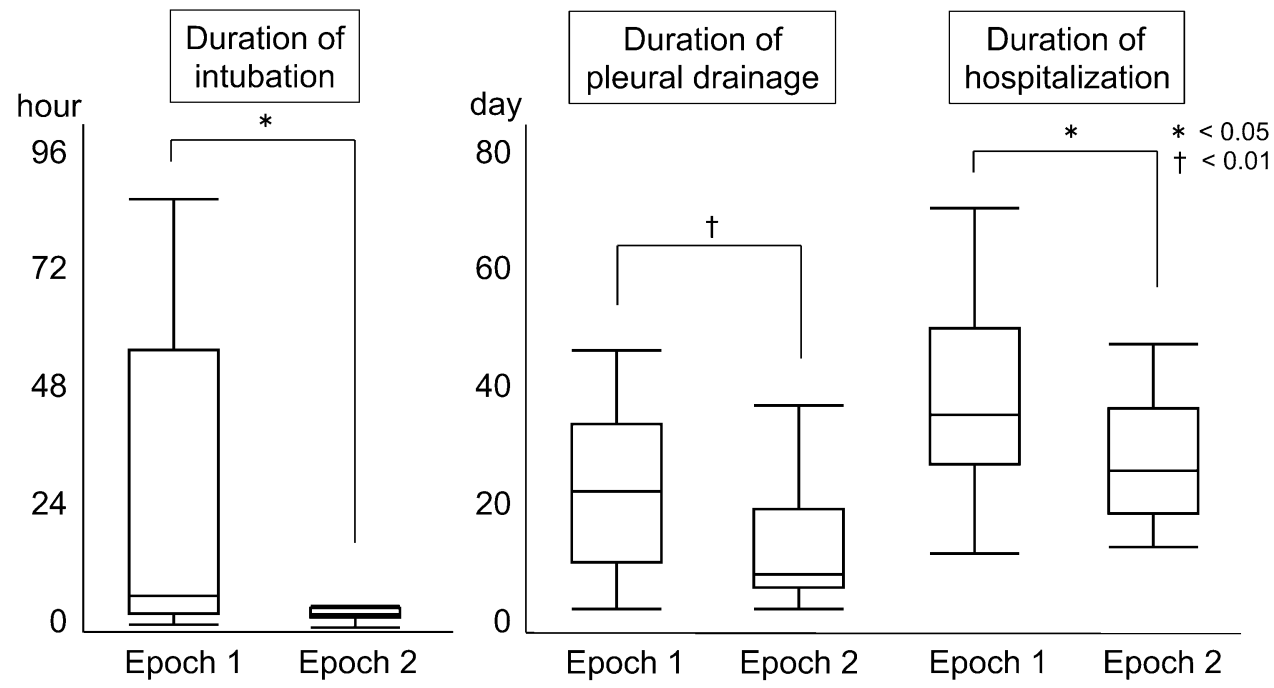

Epoch 1: extubation after weaning of NO therapy Epoch 2: HFNC-iNO therapy after extubation

\section{Discussion}

We found that, since the introduction of HFNC-iNO therapy in the postoperative management of the Fontan operation in our hospital, the duration of postoperative intubation, pleural drainage time, and postoperative hospitalization decreased significantly. Avoiding prolonged intubation and positive pressure ventilation by allowing spontaneous breathing helps prevent the deleterious effects of positive intrathoracic pressure on pulmonary blood flow and hemodynamics. Moreover, HFNC-iNO therapy enabled us to extubate the patients before weaning them off iNO therapy, thus avoiding the 'rebound' effect commonly associated with extubation and iNO discontinuation. We believe that early extubation and HFNC-iNO therapy contributed to achieving stable hemodynamics in the early postoperative phase and helped to maintain low Fontan pressure, resulting in significantly greater urinary output at $6 \mathrm{~h}$ post-surgery, as well as lower infusion volume at $24 \mathrm{~h}$ post-surgery. In addition to stable hemodynamics, patients enjoyed an earlier introduction of enteral feeding and oral administration of medications for pulmonary hypertension. The rate of re-intubation after the 
Table 3 Postoperative outcomes

\begin{tabular}{|c|c|c|c|}
\hline Outcome & Epoch $1(n=24)$ & Epoch $2(n=14)$ & $p$ value \\
\hline \multicolumn{4}{|l|}{ At ICU admission } \\
\hline Mean PAP (mmHg) & $14.8 \pm 1.7$ & $14.4 \pm 1.5$ & 0.47 \\
\hline Lactate $(\mathrm{mmol} / \mathrm{L})$ & $4.0 \pm 2.3$ & $3.8 \pm 1.4$ & 0.67 \\
\hline Creatinine $(\mathrm{mg} / \mathrm{dL})$ & $0.34 \pm 0.09$ & $0.32 \pm 0.11$ & 0.25 \\
\hline $\mathrm{P} / \mathrm{F}$ ratio & $234 \pm 109$ & $243 \pm 160$ & 0.84 \\
\hline $\mathrm{PaCO}_{2}(\mathrm{mmHg})$ & $39 \pm 5$ & $40 \pm 7$ & 0.53 \\
\hline $\mathrm{SvO}_{2}(\%)$ & $63 \pm 14$ & $57 \pm 11$ & 0.14 \\
\hline Duration of intubation (h) & $7.2[3.7-49]$ & $3.5[3.0-4.6]$ & 0.033 \\
\hline$<6$ & 11 & 12 & 0.015 \\
\hline$>24$ & 8 & 0 & 0.017 \\
\hline iNO therapy started in ICU & 7 & 4 & 0.52 \\
\hline HFNC-iNO therapy & - & 14 & - \\
\hline Re-intubation & 2 & 0 & - \\
\hline Restarted HFNC-iNO therapy instead of re-intubation & - & 7 & - \\
\hline \multicolumn{4}{|l|}{ Catecholamine score } \\
\hline At ICU admission & $8.4 \pm 3.9$ & $11.3 \pm 4.6$ & 0.04 \\
\hline 24-h score & $6.5 \pm 4.9$ & $5.2 \pm 2.1$ & 0.35 \\
\hline \multicolumn{4}{|l|}{ Urine output (mL/kg/h) } \\
\hline At $6 \mathrm{~h}$ & $2.0 \pm 1.4$ & $2.9 \pm 1.3$ & 0.042 \\
\hline At $24 \mathrm{~h}$ & $2.8 \pm 1.1$ & $2.9 \pm 1.0$ & 0.76 \\
\hline Infusion volume in $24 \mathrm{~h}(\mathrm{~mL} / \mathrm{kg})$ & $165 \pm 80$ & $110 \pm 30$ & $<0.01$ \\
\hline Postoperative intervention & 4 & 2 & 0.85 \\
\hline Postoperative infection & 4 & 2 & 0.85 \\
\hline Pleural drainage time (days) & $23[13-34]$ & $9.5[8.3-18]$ & 0.007 \\
\hline Chylothorax & 7 & 1 & 0.22 \\
\hline Length of postoperative hospitalization (days) & 36 [29-49] & $27[22-36]$ & 0.017 \\
\hline
\end{tabular}

Continuous data are presented as the mean \pm standard deviation or median [interquartile range], while categorical data are given as the number of observations. $I C U$ intensive care unit, $P A P$ pulmonary artery pressure, $\mathrm{P} / \mathrm{F}$ ratio ratio of arterial oxygen partial pressure to fractional inspired oxygen, $\mathrm{PaCO}_{2}$ partial pressure of carbon dioxide in arterial blood, $\mathrm{SvO}_{2}$ mixed venous oxygen saturation, $i N O$ inhaled nitric oxide, $H F N C$ high-flow nasal cannula

Fontan operation is reported at $4.1-14.2 \%$ [11-14], which corresponds to the rate we noted in Epoch 1. Conversely, no patients treated after the introduction of HFNC-iNO therapy required re-intubation. In Epoch 2, seven patients had hemodynamic insufficiency after extubation and iNO discontinuation but achieved hemodynamic stability by restarting HFNC-iNO therapy, without the need for reintubation. As previously reported [11-16], early extubation, lower postoperative infusion volume, and a lack of need for re-intubation likely account for the shorter duration of pleural drainage and postoperative hospitalization noted in Epoch 2. There were no significant between-group differences in other factors associated with the duration of pleural drainage and postoperative hospitalization, such as hypoplastic left heart syndrome, high pulmonary artery pressure, modified ultrafiltration, fenestration, postoperative infection, chylothorax, and postoperative intervention $[14,15,17,18]$.
To the best of our knowledge, there is no report about the usefulness of HFNC-iNO after surgery for congenital heart disease, especially in Fontan surgery. Several reports have described iNO therapy delivered via the HFNC in infants with pulmonary hypertension and respiratory distress [8, 9]. Although the administration of phosphodiesterase $\mathrm{V}$ inhibitor via the nasogastric tube was reported for managing postoperative pulmonary hypertension in congenital heart disease and preventing the "rebound" phenomenon, this approach is associated with adverse effects on systemic hemodynamics and an unpredictable degree of gastrointestinal absorption in the acute postoperative period. Furthermore, a randomized study by Stocker et al. reported that intravenous sildenafil significantly reduced systemic blood pressure [7]. Nemoto et al. also reported that a significant decrease in blood pressure was observed in $10 \%$ of patients after sildenafil administration, and that oral administration of sildenafil required several hours to decrease pulmonary 
artery pressure [6]. In contrast, our method involving postextubation HFNC-iNO therapy decreased pulmonary artery pressure and central venous pressure within minutes and, moreover, did not appear to be associated with adverse effects in terms of systemic hemodynamics.

HFNC oxygen therapy delivers heated and humidified oxygen at slight positive end-expiratory pressure, which can improve alveolar recruitment, oxygenation, $\mathrm{CO}_{2}$ clearance, and lung compliance, thereby decreasing the work of breathing, as well as the need for intubation in patients with respiratory distress $[19,20]$. Furthermore, HFNC oxygen therapy significantly improves the response to iNO [21]. In our practice, we used HFNC oxygen therapy at a flow rate of $2 \mathrm{~L} / \mathrm{kg} / \mathrm{min}$, which is well above the usual inspiratory and expiratory flow rate in children, allowing us to achieve a tracheal NO concentration close to the target concentration. DiBlasi et al. reported a difference of approximately $20 \%$ between measured tracheal NO levels and the target NO concentration when using HFNC-iNO therapy at a flow rate of $2 \mathrm{~L} / \mathrm{kg} / \mathrm{min}$ [22].

Several aspects of HFNC-iNO therapy require special attention. Since NO itself flows continuously and there is no gas discharge system within the HFNC-iNO circuit itself, it is necessary to carefully consider the patient's safety, as well as the influence of NO leaks on medical personnel and other patients in the vicinity. A previous study in our institute determined that the $\mathrm{NO}_{2}$ concentration in our ICU while using HFNC-iNO therapy is $0.010-0.018 \mathrm{ppm}$, which is below Japan's environmental standards (0.04-0.06 ppm) [23]. Additionally, patients should be closely monitored for symptoms of the 'rebound' phenomenon caused by temporary interruption of oxygen and NO due to accidental disconnection of the HFNC. Therefore, the use of HFNCiNO therapy is recommended in ICUs with appropriate air ventilation equipment and manned by an exclusive staff of doctors and nurses.

One important advantage of HFNC-iNO therapy is its wide indication. In addition to Fontan patients, HFNC-iNO therapy is likely to be indicated even in other congenital heart diseases with postoperative pulmonary hypertension, such as tetralogy of Fallot with pulmonary atresia and major aortopulmonary collateral arteries.

\section{Study Limitations}

This study is limited by its retrospective, single-center design and small sample size (number of patients in each group). We stratified the patients into groups according to the date HFNC-iNO therapy was introduced in our hospital, in January 2014. Although our surgical and perioperative management protocols other than HFNC-iNO therapy did not differ between Epoch 1 and Epoch 2, time-related bias cannot be excluded. Furthermore, we could not exclude the possibility that, in Epoch 2, the decision to extubate might have been issued sooner on account of the fact that HFNCiNO therapy could be provided as a rescue strategy if the patient developed hemodynamic instability after extubation. Finally, because the decision to start and discontinue iNO therapy in the operating room and ICU was based on the agreement and clinical judgment of the attending surgeon, the anesthesiologist, and the intensivists, it is difficult to quantify the effect of selection bias.

Therefore, future prospective and multi-center studies with a larger sample are warranted to validate our present conclusions regarding the efficacy of post-extubation HFNCiNO therapy.

\section{Conclusion}

Our results suggest that high-flow nasal oxygen therapy with iNO reduces the time required for postoperative intubation, drainage, and hospitalization in the postoperative management of patients with unstable hemodynamics after the Fontan operation.

Acknowledgements The authors wish to acknowledge the contributions of Masahiro Sawatake and Akihito Inoue for setup, maintenance, and modification of the HFNC-iNO therapy circuit, and would like to thank Editage (www.editage.jp) for English language editing.

\section{Compliance with Ethical Standards}

Conflict of interest The authors declare that they have no competing interests.

Research Involving Human Participants and/or Animals Permission to conduct this retrospective study and publish the outcomes was provided by the Institutional Review Board of our institution.

Informed Consent Written informed consent for the use of patient records was obtained from the legal guardian of each patient.

\section{References}

1. Checchia PA, Bronicki RA (2012) Review of inhaled nitric oxide in the pediatric cardiac surgery setting. Pediatr Cardiol 33:493505. https://doi.org/10.1007/s00246-012-0172-4

2. Gamillscheg A, Zobel G, Urlesberger B, Berger J, Dacar D, Stein JI, Rigler B, Metzler H, Beitzke A (1997) Inhaled nitric oxide in patients with critical pulmonary perfusion after Fontan-type procedures and bidirectional Glenn anastomosis. J Thorac Cardiovasc Surg 113:435-442. https://doi.org/10.1016/S0022-5223(97)70355 $-6$

3. Agarwel HS, Churchwell KB, Doyle TP, Christian KG, Drinkwater DC Jr, Byrne DW, Taylor MB (2006) Inhaled nitric oxide use in bidirectional Glenn anastomosis for elevated Glenn pressures. Ann Thorac Surg 81:1429-1435. https://doi.org/10.1016/j.athor acsur.2005.11.004 
4. Cai J, Su Z, Shi Z, Zhou Y, Xu Z, Xu Z, Yang Y (2008) Nitric oxide and milrinone: combined effect on pulmonary circulation after Fontan-type procedure: a prospective, randomized study. Ann Thorac Surg 86:882-888. https://doi.org/10.1016/j.athor acsur.2008.05.014

5. Frostell C, Fratacci MD, Wain JC, Jones R, Zapol WM (1991) Inhaled nitric oxide: a selective pulmonary vasodilator reversing hypoxic pulmonary vasoconstriction. Circulation 83:2038-2047

6. Nemoto S, Sasaki T, Ozawa H, Katsumata T, Kishi K, Okumura K, Mori Y, Umegaki O (2010) Oral sildenafil for persistent pulmonary hypertension early after congenital cardiac surgery in children. Eur J Caridiothorac Surg 38:71-77. https://doi. org/10.1016/j.ejcts.2010.01.045

7. Stocker C, Penny DJ, Brizard CP, Cochrane AD, Soto R, Shekerdemian LS (2003) Intravenous sildenafil and inhaled nitric oxide: a randomised trial in infants after cardiac surgery. Intensive Care Med 29:1996-2003. https://doi.org/10.1007/s00134-003-2016-4

8. Ismail A, Sharara-Chami R, El-Khatib M (2014) Combination of high-flow nasal cannula oxygen therapy and inhaled nitric oxide in a paediatric patient with respiratory distress. Anaesth Intensive Care 42:521-523

9. Lindwell R, Blennow M, Svensson M, Jonsson B, BerggrenBoström E, Flanby M, Lönnqvist PA, Frostell C, Norman M (2005) A pilot study of inhaled nitric oxide in preterm infants treated with nasal continuous positive airway pressure for respiratory distress syndrome. Intensive Care Med 31:959-964. https:// doi.org/10.1007/s00134-005-2593-5

10. Nakata S, Imai Y, Takanashi Y, Kurosawa H, Tezuka K, Nakazawa M, Ando M, Takao A (1984) A new method for the quantitative standardization of cross-sectional areas of the pulmonary arteries in congenital heart diseases with decreased pulmonary blood flow. J Thorac Cardiovasc Surg 88:610-619

11. Gupta P, Rettinganti M, Gossett JM, Yeh JC, Jeffries HE, Rice TB, Wetzel RC (2016) Risk factors for mechanical ventilation and reintubation after pediatric heart surgery. J Thorac Cardiovasc Surg 151:451-458. https://doi.org/10.1016/j.jtcvs.2015.09.080

12. Mahle WT, Jacobs JP, Jacobs ML, Kim S, Kirshbom PM, Pasquali SK, Austin EH, Kanter KR, Nicolson SC, Hill KD (2016) Early extubation after repair of tetralogy of Fallot and the Fontan procedure: an analysis of the Society of Thoracic Surgeons Congenital Heart Surgery Database. Ann Thorac Surg 102:850-858. https:// doi.org/10.1016/j.athoracsur.2016.03.013

13. Mutsuga M, Quinonez LG, Mackie AS, Norris CM, Marchak BE, Rutledge JM, Rebeyka IM, Ross DB (2012) Fast-track extubation after modified Fontan procedure. J Thorac Cardiovasc Surg 144:547-552. https://doi.org/10.1016/j.jtcvs.2012.05.047

14. Sasaki J, Dykes JC, Sosa LJ, Salvaggio JL, Tablante MD, Ojito J, Khan DM, Hannan RL, Rossi AF, Burke RP, Wernovsky G (2016) Risk factors for longer hospital stay following the
Fontan operation. Pediatr Crit Care Med 17:411-419. https://doi. org/10.1097/PCC.0000000000000701

15. Salvin JW, Scheurer MA, Laussen PC, Mayer JE Jr, Del Nido PJ, Pigula FA, Bacha EA, Thiagarajan RR (2008) Factors associated with prolonged recovery after the Fontan operation. Circulation 118:S171-S176. https://doi.org/10.1161/CIRCULATIONAHA

16. Alghamdi AA, Singh SK, Hamilton BC, Yadava M, Holtby H, Van Arsdell GS, Al-Radi OO (2010) Early extubation after pediatric cardiac surgery: systematic review, meta-analysis, and evidencebased recommendations. J Card Surg 25:586-595. https://doi.org /10.1111/j.1540-8191.2010.01088.x

17. Rogers LS, Glatz AC, Ravishankar C, Spray TL, Nicolson SC, Rychik J, Rush CH, Gaynor JW, Goldberg DJ (2012) 18 years of the Fontan operation at a single institution: results from 771 consecutive patients. J Am Coll Cardiol 60:1018-1025. https:// doi.org/10.1016/j.jacc.2012.05.010

18. Iyengar AJ, Winlaw DS, Galati JC, Celermajer DS, Wheaton GR, Gentles TL, Grigg LE, Weintraub RG, Bullock A, Justo RN, d'Udekem Y (2014) Trends in Fontan surgery and risk factors for early adverse outcomes after Fontan surgery: The Australia and New Zealand Fontan Registry experience. J Thorac Cardiovasc Surg 148:566-575. https://doi.org/10.1016/j.jtcvs.2013.09.074

19. Parke R, McGuinness S, Eccleston M (2009) Nasal high-flow therapy delivers low level positive airway pressure. Br J Anaesth 103:886-890. https://doi.org/10.1093/bja/aep280

20. Dysart K, Miller TL, Wolfson MR, Shaffer TH (2009) Research in high flow therapy: mechanisms of action. Respir Med 103:1400 1405. https://doi.org/10.1016/j.rmed.2009.04.007

21. Johanningman JA, Davis K, Campbell RS, Luchette FA, Frame SB, Branson RD (2000) Positive end-expiratory pressure and response to inhaled nitric oxide: changing nonresponders to responders. Surgery 127:390-394. https://doi.org/10.1067/ msy.2000.104117

22. DiBlasi RM, Dupras D, Kearney C, Costa E, Griebel JL (2015) Nitric oxide delivery by neonatal noninvasive respiratory support devices. Respir Care 60:219-230. https://doi.org/10.4187/respc are. 03278

23. Kyogoku M, Takeuchi M, Tachibana K, Shimizu Y, Hatachi T, Moon K, Isaka K, Fujiwara F (2017) Efficacy and safety of inhaled nitric oxide using nasal high-flow oxygen therapy after pediatric cardiac surgery for congenital heart disease. J Jpn Soc Intensive Care Med 24:14-17. https://doi.org/10.3918/jsicm.24_14

Publisher's Note Springer Nature remains neutral with regard to jurisdictional claims in published maps and institutional affiliations. 